\title{
Comparing the validity of trait estimates from the multidimensional forced-choice format and the rating scale format
}

\author{
Eunike Wetzel ${ }^{1,2,3} \&$ Susanne Frick ${ }^{1,2}$ \\ ${ }^{1}$ University of Konstanz \\ ${ }^{2}$ University of Mannheim \\ ${ }^{3}$ University of Vienna
}

Accepted version of article in press at Psychological Assessment

C 2019, American Psychological Association. This paper is not the copy of record and may not exactly replicate the final, authoritative version of the article. Please do not copy or cite without authors' permission. The final article will be available, upon publication, via its DOI: $10.1037 /$ pas0000781

Date of acceptance: September 25, 2019

Author note:

Eunike Wetzel is now at Otto-von-Guericke University Magdeburg. This research was supported by a grant from the German Research Foundation (DFG) to Eunike Wetzel (WE 5586/2-1) as well as by the Elite Program for Postdocs of the Baden-Württemberg Stiftung and the Young Scholar Fund of the University of Konstanz. We thank Joschka Cremers, 
Theresa Falter, Celia Fürst, Veronika Held, Lars Hilbert, Clara Jupe, Mara Nuttelmann, Rachelle Sass, and Georg Schäfer for their help with data collection. We thank Michael Dantlgraber for allowing us to use the items from his Master's thesis.

Correspondence concerning this article should be addressed to Eunike Wetzel, Department of Psychology, Otto-von-Guericke University Magdeburg, Universitätsplatz 2, 39106 Magdeburg, Germany.

The following material is available on the Open Science Framework:

Pre-registration: https://osf.io/m78fx

Data: https://osf.io/z9w6s/ 


\title{
Comparing the validity of trait estimates from the multidimensional forced-choice format and the rating scale format
}

\begin{abstract}
The multidimensional forced-choice (MFC) format has been proposed as an alternative to rating scales (RS) that may be less susceptible to response biases. The goal of this study was to compare the validity of trait estimates from the MFC and the RS format when using normative scoring for both formats. We focused on construct validity and criterion-related validity. In addition, we investigated test-retest reliability over a period of six months. Participants were randomly assigned the MFC $(N=593)$ or the $\mathrm{RS}(N=622)$ version of the Big Five Triplets. In addition to self-ratings on the Big Five Triplets and other personality questionnaires and criteria, we also obtained other-ratings $(N=770)$ for the Big Five Triplets. The Big Five in the Big Five Triplets corresponded well with the Big Five in the Big Five Inventory except for agreeableness in the MFC version. The majority of the construct validity coefficients differed between the MFC and the RS version whereas criterion-related validities were very similar. The self- and other-rated Big Five Triplets showed higher correlations in the MFC format than in the RS format. The reliability of test scores on the Big Five and testretest reliabilities were lower for MFC compared to RS. For the MFC format to be able to replace the RS format, more research on how to obtain ideal constellations of items that are matched in their desirability is needed.
\end{abstract}

Keywords: multidimensional forced-choice; rating scale; response format; item format; Thurstonian item response model

Public significance statement: The response format used in a questionnaire can influence inferences on respondents' trait levels and therefore diagnostic decisions. This study finds that 
the multidimensional forced-choice version of a Big Five questionnaire, in which participants rank items presented in triplets, overall measures the same constructs and is related to criteria (e.g., number of Facebook friends) in the same way as a rating scale version of the questionnaire, though relations with other traits tend to differ in magnitude. 
In many contexts, the psychological assessment of personality traits, attitudes, and other constructs is based on self-report questionnaires. For example, the five trait domain qualifiers in the ICD-11's dimensional trait model for the classification of personality disorders (World Health Organization, 2018) are assessed with self-report questionnaires (Oltmanns \& Widiger, 2018). Most self-report questionnaires require participants to rate themselves regarding the presented statements on a rating scale with multiple response categories, such as strongly disagree - disagree - neutral - agree - strongly agree. A multitude of research has shown that responses given in the rating scale (RS) format are susceptible to a number of response biases, including response styles (e.g., extreme response style, acquiescence) and socially desirable responding or explicit faking (for an overview see Van Vaerenbergh \& Thomas, 2013; Wetzel, Böhnke, \& Brown, 2016). Concerns about the effects of these response biases on data quality and inferences on individuals' trait levels have led researchers to propose using alternative item and response formats that might be less prone to these biases. One such alternative that has been proposed is the forced-choice format (e.g., Brogden, 1954; Sisson, 1948). In the forced-choice format, multiple items are presented simultaneously and participants have to rank the items according to how well they describe them or select the item that is most (and/or least) like them (see Figure 1 for an example). These items can all measure the same construct or measure different constructs. In this paper, we are only concerned with the latter, the multidimensional forced-choice (MFC) format.

By nature, MFC data are relative. Whether absolute trait levels can be derived from this type of data depends on the scoring approach and on the questionnaire design. There are two general approaches to scoring MFC data: 1) ipsative scoring and 2) normative scoring. Ipsative scores result when the total score is a constant across persons (Clemans, 1966). This is the case when MFC data are scored using classical test theory (sum scores or mean scores) or using unidimensional item response theory (IRT) models, though some strategies for obtaining partially ipsative scores have been developed such as combining positively and 
negatively keyed items in a block or adding filler items which are not analyzed (Heggestad, Morrison, Reeve, \& McCloy, 2006). Ipsative scores are only comparable within persons (Hicks, 1970) and mathematically imply distorted trait intercorrelations and validities (Clemans, 1966). On the other hand, normative scores can be obtained from IRT models that explicitly model the relative nature of item responses within blocks. A number of IRT models has been developed for forced-choice data, including the Thurstonian IRT model (Brown \& Maydeu-Olivares, 2011), the multi-unidimensional pairwise-preference model (Stark, Chernyshenko, \& Drasgow, 2005) and the multidimensional unfolding approach (McCloy, Heggestad, \& Reeve, 2005). For an overview and a common framework for these IRT models see Brown (2016). Normative trait estimates allow drawing interindividual comparisons, which is needed in many psychological assessment settings such as selection contexts (e.g., personnel selection). Furthermore, they allow comparing the validity of MFC trait estimates with that of RS trait estimates, which are always normative, unconfounded by mathematical constraints. Therefore, in this paper, we use the Thurstonian IRT model to obtain normative scores from MFC data ${ }^{1}$.

\section{Previous research comparing the MFC and the RS format}

In order to evaluate the MFC format, it should be compared to the current standard, rating scales. Several previous studies have compared the MFC format and the RS format with respect to aspects relevant to testing and assessment. For example, several studies found that less faking occurred in the MFC format compared with the RS format at the group level (Christiansen, Burns, \& Montgomery, 2005; Heggestad et al., 2006; Jackson, Wroblewski, \& Ashton, 2000), though one study found that similar levels of faking occurred at the individual

\footnotetext{
${ }^{1}$ The Thurstonian IRT model is the most appropriate model for our analyses because we administered items that assume a dominance response process while most other IRT models developed for forced-choice data assume ideal point items. Furthermore, the Thurstonian IRT model currently appears to be the only model for which item parameters can readily be estimated in popular software programs.
} 
level (Heggestad et al., 2006). A recent meta-analysis found that the overall effect size for faking of FC measures was $d=0.06$ (Cao \& Drasgow, 2019), which is substantially smaller than the average effects size reported in a meta-analysis of faking of RS measures $(d=0.26$; Birkeland, Manson, Kisamore, Brannick, \& Smith, 2006). In addition, Sass, Frick, Reips, and Wetzel (2018) did not find any significant differences in the self-reported test motivation of participants between four different variants of the MFC format (pairs, triplets, quads, pentads) and the RS format.

A number of studies compared the two formats with respect to their criterion-related validity. For example, Bartram (2007) showed that the forced-choice version of the Occupational Personality Questionnaire (OPQ; Saville, Holdsworth, Nyfield, Cramp, \& Mabey, 1993) yielded higher criterion-related validities than the RS version for predicting performance ratings across a number of occupations. A meta-analysis by Salgado and Táuriz (2014) found that the validity of Big Five scores from forced-choice measures for predicting academic and job performance was similar to or larger than the validity of Big Five scores from RS measures. However, these previous comparisons between the MFC and RS format were based on ipsative or partially ipsative scoring for MFC and normative scoring for RS. Thus, they confounded response format and scoring approach. A few more recent studies overcame this limitation by applying Thurstonian item response modeling to investigate validity-related issues ${ }^{2}$. Brown and Maydeu-Olivares (2011) found similar intercorrelations among the Big Five for an MFC version scored with the Thurstonian IRT model and an RS version of an instrument created from the International Personality Item Pool Big Five Factor Markers (Goldberg, 1992). Brown and Maydeu-Olivares (2013) compared classical and IRT scoring of the Customer Contact Styles Questionnaire (SHL, 1997), a questionnaire that

\footnotetext{
${ }^{2}$ We only summarize studies that applied Thurstonian item response modeling in the following because this is the model we also applied. For an investigation of validity-related issues in the context of an instrument with ideal point items using the multi-unidimensional pairwise preference model see Zhang et al. (2019).
} 
employs a combined MFC and RS format in which each of the four items in a block are rated individually and participants additionally select the two items that are most and least like them. The authors discovered that the Thurstonian IRT-scored MFC data yielded similar construct and criterion-related validity as the classically and IRT-scored RS data, whereas the classically scored MFC data showed clear signs of ipsativity (distorted individual profiles with mean scores around 0 , a negative average intercorrelation among the 16 traits, distorted correlations with other traits). Similarly, Lee, Lee, and Stark (2018) compared Thurstonian IRT normative scoring of MFC data from a Big Five instrument to two partially ipsative methods (classical test theory and unidimensional IRT) with respect to construct validity and criterion-related validity. They found that Thurstonian IRT scoring achieved slightly better construct and criterion-related validities than the other two scoring approaches. Further, they found similar results between Thurstonian IRT scoring of MFC data and classical scoring of RS data. Lastly, Guenole, Brown, and Cooper (2018) found promising results for the application of Thurstonian item response modeling to the assessment of work-related maladaptive personality traits using the forced-choice format.

\section{The present study}

In this study, we applied normative scoring for the MFC format, allowing a fairer comparison between the MFC and RS formats based on the same type of data. Thus, the goal of this study was to compare the validity of trait estimates from the MFC and the RS format when using normative scoring for both formats. We focused on construct validity and criterion-related validity. In addition, we investigated test-retest reliability over a period of six months. This study extends previous research in several important ways: First, we compared the MFC and RS format by consistently using normative scoring, while a lot of previous research confounded normative and ipsative (or partially ipsative) scoring. Second, we correlated the MFC or RS version of our instrument with other questionnaires with the same format (e.g., an MFC Big Five questionnaire correlated with an MFC HEXACO questionnaire 
and vice versa for RS). This is an improvement over previous research investigating construct validity. Previous research only used RS instruments for validation and thereby potentially made the comparison between formats unbalanced because common method variance due to shared response format only existed on the part of RS. Third, this is the first study that also obtained other-ratings. We investigated validity by correlating self-ratings with other-ratings, thus eliminating the influence of common method bias. Fourth, this study used a betweensubjects design to avoid cross-over effects between the MFC and RS versions of the questionnaire, which may have occurred in previous research that exclusively employed within-subjects designs. Fifth, we used a wide range of criteria assessed with different methods (e.g., frequency of behaviors, intelligence test score, objective measurement of punctuality), while previous research mainly relied on criteria assessed with RS. Sixth, this is the first study that also investigated test-retest reliability using Thurstonian item response modeling for the MFC format. In addition, this study is the first one to investigate this topic using preregistered hypotheses. Thus, this study provides a comprehensive investigation of the validity of trait estimates from the MFC format and the RS format. The results of our study can inform researchers and practitioners regarding the feasibility of applying the MFC format.

\section{Hypotheses}

We investigated construct validity in the framework of a multitrait-multimethod (MTMM) analysis (Campbell \& Fiske, 1959). That is, we compared monotrait-heteromethod correlations (e.g., neuroticism in the MFC format with neuroticism in the RS format) with heterotrait-monomethod correlations (e.g., neuroticism in the MFC format with extraversion in the MFC format) and heterotrait-heteromethod correlations (e.g., neuroticism in the MFC format with extraversion in the RS format). However, our analyses differ somewhat from classical MTMM designs because we focus on the comparison between MFC and RS, which consisted of data from independent groups. More precisely, we compared the magnitude of 
different correlations across MFC and RS groups. For example, we compared whether the monotrait-heteromethod correlation between openness in the Big Five Triplets and openness in the HEXACO is similar or differs for MFC versus RS. Note further that mono- versus heteromethod for some hypotheses refers to self- and other-ratings in addition to the response format. Hypotheses 1 to 4 address convergent validity (i.e., monotrait) aspects of the MTMM analysis and hypotheses 5 and 6 address discriminant validity (i.e., heterotrait) aspects of the MTMM analysis. In general, we expected similar construct validity for MFC and RS trait estimates on the Big Five Triplets (BFT; see below) when the two constructs being correlated were assessed with the same questionnaire format. Furthermore, based on previous research, we expected higher criterion-related validities for MFC trait estimates. We pre-registered the following hypotheses on the Open Science Framework (https://osf.io/m78fx):

\section{Construct validity: Convergent validity}

H1: MFC trait estimates on the Big Five (from BFT-MFC) and RS trait estimates on the Big Five (from the Big Five Inventory) will correlate strongly $(>.50)$, but not perfectly $(<1)$. H2: The correlations of RS trait estimates on the Big Five from BFT-RS and the Big Five Inventory will be higher than the correlations in $\mathrm{H} 1$.

H3: MFC trait estimates will show similar correlations as RS trait estimates on the same or similar constructs when the response format is the same (e.g., BFT-MFC self with HEXACOMFC self compared to BFT-RS self with HEXACO-RS self or BFT-MFC self with BFTMFC other compared to BFT-RS self with BFT-RS other).

H4: MFC trait estimates will show similar correlations as RS trait estimates on the same or similar constructs when the response format is not the same (e.g., BFT-MFC self with HEXACO-RS self or BFT-MFC self with BFT-RS other), but these will be lower compared to those in $\mathrm{H} 3$.

\section{Construct validity: Discriminant validity}


H5: MFC trait estimates will show similar discriminant validities as RS trait estimates on dissimilar constructs when the response format is the same (e.g., BFT-MFC self with HEXACO-MFC self compared to BFT-RS self with HEXACO-RS self).

H6: MFC trait estimates will show lower discriminant validities than RS trait estimates on dissimilar constructs when the response format only matches for the RS trait estimates (e.g., BFT-MFC self with Short Dark Triad self compared to BFT-RS self with Short Dark Triad self).

\section{Criterion-related validity}

H7: MFC trait estimates will on average show higher criterion-related validities than RS trait estimates.

\section{Method}

The comparison of the MFC format and the RS format regarding validity was conducted with a newly developed Big Five instrument, the Big Five Triplets. In the following, we describe the development of the Big Five Triplets. Then, we describe the study design, sample, measures, and analyses. The study design and analysis plan were preregistered (https://osf.io/m78fx). The item-level data for the personality questionnaires are available from https://osf.io/z9w6s/. This study was exempt from approval by an ethics committee.

\section{The Big Five Triplets}

For the purposes of this research project, we constructed an MFC questionnaire for the assessment of the Big Five following the recommendations detailed in Brown and MaydeuOlivares (2011). The resulting instrument, the BFT, presents items in triplets with a full ranking task (see sample triplet in Figure 1). We summarize the test construction process here and refer interested readers to a more detailed description available from https://osf.io/ft9ud/. We started with an item pool of 213 items, which were taken from the International Personality Item Pool (https://ipip.ori.org; Goldberg et al., 2006) and a Master's thesis 
(Dantlgraber, 2011) or constructed by the authors. All items in this initial item pool were rated regarding their social desirability by 33 psychology students on a five-point rating scale with the categories socially undesirable, slightly socially undesirable, neutral, slightly socially desirable, and socially desirable. Using the median social desirability ratings across raters, we then constructed a pilot version of the instrument with 71 triplets in which most triplets (48) were matched regarding their social desirability (i.e., items presented in a triplet were of the same social desirability category). The remaining 23 triplets contained items from neighboring social desirability categories (e.g., two neutral items and one slightly socially undesirable item).

We used data from an MTurk sample with $N=1,006$ to reduce the number of triplets to 33. We analyzed the data using the Thurstonian IRT model (Brown \& Maydeu-Olivares, 2011, 2013) and considered standardized factor loadings, item informations, and correlations between items for selecting items. We collected data on the revised instrument using Prolific Academic $(N=1,286)$ and conducted the same analyses, arriving at the final version of the BFT with 20 triplets, all of which are matched regarding their social desirability. Because we prioritized the desirability matching and constructing triplets that contain both positively and negatively keyed items, balancing the number of items per trait was not possible. The BFT thus consists of 16 neuroticism items, 13 extraversion items, 10 openness items, seven agreeableness items, and 14 conscientiousness items. The BFT is available from https://osf.io/ft9ud/.

\section{Study design}

Participants were randomly assigned to one of three response format groups: MFCoriginal $(N=593)$, MFC-altered $(N=652)$, and RS $(N=622)$. The MFC-original group filled out the original version of the BFT described above. The MFC-altered group filled out an altered version of the BFT for a study on faking. Their data will not be analyzed in this study. Therefore, we will refer to the MFC-original group simply as the MFC group. The RS group 
filled out the items from the BFT with a four-point rating scale (strongly disagree, disagree, agree, strongly agree). Participants first filled out the BFT in the respective format, then they responded to a number of questions relating to social activities, health, abilities, and other variables (see criteria below). Next, participants filled out other personality questionnaires (see measures below). Then, they received a fake-good instruction and filled out the BFT again. This part of the data collection is not relevant to the current study. After the end of the survey, participants in the laboratory subsample could optionally take a short intelligence test. Lastly, they were debriefed and participants in the laboratory subsample were additionally asked whether they would provide contact information for one or two persons close to them for the purpose of obtaining other-ratings of their personality and whether they would consent to being contacted for a second wave of data collection.

Other-raters were invited via email to participate in an online survey about one week after the target person's laboratory session. Approximately six months after the laboratory session, participants who had given their consent to being contacted for a second wave were sent an email invitation to take part in an online survey.

\section{Sample}

The sample consisted of two subsamples: one laboratory sample and one Internet access panel sample. The laboratory sample consisted of 1,042 persons (across all three response format groups) from two German universities. Of these 1,042 persons, three participants were removed because they filled out the survey in less than $2 \mathrm{SD}$ of the average completion time of the participants. Of the remaining participants, 117 were removed because they responded incorrectly to one or both instructed response items, one participant was removed because he or she did not read the instructions, and four participants were removed because they exhibited language difficulties. Furthermore, seven participants took part twice and their second participation was removed. Thus, the final sample size of the laboratory study was $910\left(74.7 \%\right.$ female, $25.1 \%$ male, $0.2 \%$ transgender; $\left.M_{\text {age }}=22.56, S D=4.67\right)$. 
Participants in the laboratory study were remunerated either with research participation credit or money (between 8 and 15 Euros depending on the length of the session). Participants who took the intelligence test could receive feedback on their scores.

The access panel sample was collected with Respondi. Respondi participants once register to the panel and are then invited to selected studies via email. For our study, only participants who were between 18 and 30 years old and whose first language was German were invited. The age range was restricted for the purposes of a different study on faking, for which the data was collected together with this study. In total (across all three response format groups), 1,217 participants from the access panel took part. Of these, 91 were redirected without completing the study because they entered an age that was outside our specified range, they did not give German as their first language, or they participated after the quota for gender ( $50 \%$ female) was already full. In addition, we removed 56 participants who were below the time cut-off ( 2 SD below the average) and 113 participants who responded incorrectly to one or both instructed response items. Thus, the final access panel sample consisted of 957 participants $\left(54.2 \%\right.$ female, $45.5 \%$ male, $0.3 \%$ transgender; $M_{\text {age }}=24.35, S D$ =3.66). Participants in the access panel sample were remunerated with 4 Euros each.

The complete sample across all three response format groups therefore consisted of 1,867 persons. Of these, 593 participants were in the MFC group (62.7\% female, $36.9 \%$ male, $0.3 \%$ transgender; $\left.M_{\text {age }}=23.40, S D=4.09\right)$ and 622 were in the RS group $(64.7 \%$ female, $34.9 \%$ male, $0.3 \%$ transgender; $\left.M_{\text {age }}=23.64, S D=4.39\right)$.

Nine hundred eighty-nine of the participants in the laboratory subsample (including all three response format groups) were invited to the second wave approximately six months after the laboratory session. Of these, 638 took part in the T2 data collection. We removed 17 participants because their completion time was below the cut-off, 45 participants because they responded incorrectly to the instructed response item, three participants who had been reinvited mistakenly, one participant whose ID had not been recorded, and three participants 
who had received the wrong response format version. Thus, the total sample size for T2 was $569\left(77.5 \%\right.$ female, $22.3 \%$ male, $0.2 \%$ transgender; $\left.M_{\text {age }}=23.04, S D_{\text {age }}=4.63\right)$. Participants were remunerated with an Amazon voucher over eight Euros or research participation credit. Since participants in the third response format group (MFC-altered) also received the original version of the BFT at T2, more participants filled out the MFC version (378) than the RS version (191). Test-retest reliabilities were estimated with participants who had filled out the MFC(-original) or RS version of the BFT at both time points, though item parameters were estimated with all participants who had provided relevant data (i.e., including participants from the third response format group at T2 and participants who only participated in T1).

\section{Other-raters}

Participants from the laboratory subsample were asked to provide contact information for two people whom they were close with and had known for at least six months. If two other-raters were available, one was assigned the MFC version and one the RS version. If we only had contact information for one other-rater, she or he was randomly allocated one version. We sent emails to 978 potential other-raters of whom $822(84 \%)$ participated. Of these, we removed eight other-raters because their completion time was below $2 \mathrm{SD}$ of the average and 38 other-raters because they responded incorrectly to one or both instructed response items. We additionally removed data from four other-raters whose target person had been removed from the analyses. Lastly, we mistakenly had data from three other-raters for two participants and removed the respective third other-rater. Thus, we analyzed data from 770 other-raters $\left(68.3 \%\right.$ female, $31.7 \%$ male; $\left.M_{\text {age }}=27.92, S D_{\text {age }}=12.05\right)$. In total, we had data from two other-raters for 250 participants and from one other-rater for 270 participants. Other-raters could take part in a lottery of 10 Amazon vouchers worth 25, 50, 75, or 100 Euros (500 Euros in total).

\section{Measures}


Descriptive statistics for the sample on the criterion variables are shown in Table 1 and histograms or barplots for the criteria are shown in Figure S1 of the supplemental online material. Reliability coefficients for scores on the BFT and other personality scales used for the construct validity analyses are depicted in supplemental Table S1. For convergent validity, we applied another Big Five questionnaire and a HEXACO questionnaire. For discriminant validity, we additionally applied a short measure of the Dark Triad consisting of narcissism, Machiavellianism, and psychopathy as well as longer questionnaires assessing each of these three traits individually.

Big Five. The Big Five domains neuroticism, extraversion, openness to experience, agreeableness, and conscientiousness were assessed with the BFT (see above) and the Big Five Inventory (BFI; John, Donahue, \& Kentle, 1991; John \& Srivastava, 1999). Empirical reliabilities for maximum a posteriori trait estimates on the Big Five ranged from 0.68 for agreeableness to 0.84 for conscientiousness for BFT-MFC and from 0.79 for agreeableness to 0.91 for neuroticism and extraversion for BFT-RS (see Table S1).

HEXACO. We applied the HEXACO-60 (Ashton \& Lee, 2009) to assess the HEXACO dimensions honesty-humility, emotionality, extraversion, agreeableness versus anger, conscientiousness, and openness to experience. For the purposes of this study, we created an MFC version of the HEXACO-60 with triplets. At the beginning of the study, when participants were randomly assigned to the BFT-MFC group or the BFT-RS group, they were also assigned to one of the HEXACO-60 versions. Thus, approximately half of the participants in each of the response format groups received the original RS version of the HEXACO-60 and the other half received the MFC version.

Dark Triad. We applied the Short Dark Triad Scale (SD3; Jones \& Paulhus, 2014) to measure narcissism, Machiavellianism, and psychopathy. This questionnaire contains nine items for each trait (in total 27). Sample items are "It's not wise to tell your secrets" (Machiavellianism), "I have been compared to famous people" (narcissism), and "People 
often say I'm out of control" (psychopathy). Participants rated the items on a five-point rating scale ranging from strongly disagree to strongly agree.

Narcissism. Narcissism was assessed with the Narcissistic Admiration and Rivalry Questionnaire (Back et al., 2013). The Narcissistic Admiration and Rivalry Questionnaire consists of 18 items which measure two facets of narcissism with nine items each: admiration (e.g., "I am great." or "I show others how special I am.") and rivalry (e.g., "I want my rivals to fail." or "Most people won't achieve anything."). Participants rated how well the items described them on a six-point rating scale ranging from not agree at all to agree completely.

Machiavellianism. The MACH-IV Scale (Christie \& Geis, 1970) was used to assess Machiavellianism. The questionnaire contains 20 items that refer to interpersonal manipulation, cynicism, and morality. Sample items are "It is hard to get ahead without cutting corners here and there" or "The best way to handle people is to tell them what they want to hear." Participants responded on a six-point rating scale ranging from $-3=$ not agree at all to 3 = agree completely. The MACH-IV was only applied in the laboratory subsample.

Psychopathy. Psychopathy was assessed with a modified version of the Self-report Psychopathy Scale (Hare, 1985). The 31-item version we used was based on Williams, Nathanson, and Paulhus (2003). A sample item is "I have broken into a building or vehicle in order to steal something or vandalize.“ The participants responded on a five-point rating scale ranging from disagree strongly to agree strongly. The Self-report Psychopathy Scale was only applied in the laboratory subsample.

Criteria. The criterion variables can be categorized into five groups: variables related to social activities, health, charity, abilities, and other variables (see Table 1). Social activities variables consisted of how many Facebook friends participants had with the option of checking "I do not have a Facebook account." This question was analyzed as two separate variables: a dichotomous variable indicating whether participants had a Facebook account (0 $=$ no, $1=$ yes) and a continuous variable with the reported number of Facebook friends. 
Further social activities variables were the number of parties they attended a month, the number of dates they went on in a month, and the number of persons they dated per year. For their responses to the frequency questions, participants entered the respective number in a text box. Health-related variables included whether participants smoked (never - less than once a month -1 to 3 times a month - 1 to 3 times a week - on 4 to 6 days a week - every day), if every day, how many cigarettes they smoked a day (open-ended), how often they drank alcohol (never - up to once a month -2 to 4 times a month -2 to 3 times a week -4 or more times a week), whether they exercised regularly (i.e., at least once a week; yes/no), and their life satisfaction. Life satisfaction was modeled as a latent variable from the five items in the Satisfaction With Life Scale (Diener, Emmons, Larsen, \& Griffin, 1985). A sample item reads "I am satisfied with my life." Items were rated on a seven-point rating scale from strongly disagree to strongly agree.

Further, we asked participants whether they had engaged in any charity, including whether they had donated blood (yes/no), whether they had done any charity work in a social organization (yes/no), and whether they did a voluntary social year after graduating from high school (yes/no). As ability-related variables we used participants' current GPA (only for students whose major had a 1 to 4 grading system where 4 = passing) and their scores on the numeric module of the modular short intelligence test (Modularer-Kurzintelligenztest; Dantlgraber, 2015), which was used as a proxy for intelligence. The latter was modeled using a 2 parameter-logistic model. Lastly, we assessed whether participants showed up to the laboratory session on time (punctuality, yes/no), and we asked participants whether they had a job (yes/no), whether they played an instrument (yes/no), whether they engaged in any extreme sports such as paragliding (yes/no), and how many times they had traveled abroad for more than one month after graduating from high school.

For the second wave, participants filled out the same BFT version as in the first data collection. In addition, they filled out a number of personality measures including the Short 
Dark Triad Scale and the Narcissistic Admiration and Rivalry Questionnaire as well as criterion variables for the purposes of a different study. Only the BFT data from T2 will be analyzed in this study.

\section{Analyses}

Personality constructs were modeled as latent variables in the framework of IRT. We applied the Thurstonian IRT model (Brown \& Maydeu-Olivares, 2011, 2013) for MFC data and the graded response model (Samejima, 1969) for RS data. Observed scores were used to model criteria with the exception of life satisfaction and intelligence, which were modeled as latent variables. We used Mplus Version 8 (Muthén \& Muthén, 1998-2018) and the R (Team, 2013) packages MplusAutomation (Hallquist \& Wiley, 2018), psych (Revelle, 2018), Cairo (Urbanek \& Horner, 2015), and corrplot (Wei \& Simko, 2017) for data analysis.

Construct validity. Hypotheses relating to the MTMM analysis of construct validity (H1 to H6) were tested with two approaches: 1) direct model-based latent correlations and 2) correlations based on previously derived trait estimates. For the first approach, we modeled the constructs of interest with the Thurstonian IRT model or graded response model and added correlations between the different latent variables. For example, to test H1, we modeled the BFT-MFC data with the Thurstonian IRT model and the BFI data with the graded response model and added correlations between the Big Five from the BFT-MFC and the Big Five from the BFI. For the second approach, we first obtained trait estimates (maximum a posteriori estimates; MAP) from Thurstonian IRT or graded response models for the respective questionnaire (e.g., trait estimates on the Big Five from a Thurstonian IRT model of the BFT-MFC). Then, we correlated the trait estimates in a second step. For both approaches, hypotheses on similarities or differences between correlations were tested by testing the difference in the correlations' Fisher- $Z$ values for significance and by evaluating the effect size of the difference $(.10=$ small, $.30=$ moderate, $.50=$ large; Cohen, 1988). Correlations were regarded as similar ( $\mathrm{H} 3$ to $\mathrm{H} 5)$ when the difference in their Fisher- $Z$ values 
was $<|.10|$. In general, correlations based on MAP estimates can be expected to be lower than model-based correlations because measurement error is not controlled for. For the hypotheses involving other-rater data (H3 to H6), we modeled the other-raters' data in one model together with the target person's self-ratings (first approach) or correlated MAP estimates from separate models of the two data sources (second approach).

Criterion-related validity. To test $\mathrm{H} 7$ on criterion-related validity, our preregistered analysis plan indicated that we should also obtain correlations between the Big Five and our criteria. However, we realized that we had not specified in our preregistration which criteria we expected to be correlated with which Big Five domain. Including correlations which are expected to be close to zero would distort the results, as they should not differ between MFC and RS. Therefore, we asked five experts in the area of personality psychology to rate which Big Five domain they hypothesized each of the criteria would correlate with at $>|.15|$, with |.15| representing a small latent correlation according to Gignac and Szodorai (2016). Furthermore, a reviewer suggested that we look at $R^{2}$ values rather than correlations. Therefore, we added one criterion at a time to the respective model of the Big Five and estimated the regression of the criterion on the Big Five domain(s) hypothesized by the experts (e.g., we regressed the number of Facebook friends on extraversion from the BFTMFC modeled in a Thurstonian IRT model). We then computed the average $R^{2}$ value across all hypothesized relationships. We descriptively compared the average $R^{2}$ value between the MFC format and the RS format. We also provide the full correlation table between all criteria and all Big Five domains for interested readers.

Test-retest reliability. To compare the test-retest reliability of BFT-MFC and BFT-RS scores, we modeled the T1 and T2 data in latent state models based on the Thurstonian IRT model or the graded response model. That is, we constructed latent state variables for the Big Five from $\mathrm{T} 1$ and $\mathrm{T} 2$ and regressed the $\mathrm{T} 2$ variables on the $\mathrm{T} 1$ variables. These regressions 
indicate the stability and therefore test-retest reliability over a period of six months. This analysis was exploratory.

\section{Results}

Before testing our hypotheses on construct validity and criterion-related validity, we checked the intercorrelations of the Big Five in BFT-MFC and BFT-RS. For BFT-RS, intercorrelations were similar to those reported in a meta-analysis (van der Linden, Nijenhuis, \& Bakker, 2010) with the exception of some conscientiousness correlations. For example, the meta-analytic estimate of the correlation between neuroticism and conscientiousness was 0.43 whereas in the BFT-RS, the correlation was -0.02 (see Figure S2). In the BFT-MFC, there were a few more correlations that deviated from the meta-analytic estimates (see Figure S3). For example, the correlation between extraversion and conscientiousness was $-0.45(0.29$ in van der Linden et al., 2010) and the correlation between neuroticism and agreeableness was $-0.79(-0.36$ in van der Linden et al., 2010).

\section{Construct validity}

Convergent validity. Latent trait correlations on the same Big Five domain from BFTMFC and the BFI were strong for all Big Five domains (e.g., $r=.90$ for neuroticism; see Figure 2) except agreeableness $(r=.24)$. In addition, the correlation for conscientiousness was lower than would be expected if the same trait was being measured $(r=.56)$. Similar results were found for MAP-based correlations (see Table S2), though these correlations were all lower than the model-based ones, as would be expected with observed correlations. Thus, H1 was partially confirmed. For BFT-RS, monotrait correlations with the BFI ranged from .87 (conscientiousness) to .96 (neuroticism and extraversion; see Figure 2). For all Big Five domains, the BFT-RS monotrait correlation with the BFI was significantly larger than the BFT-MFC monotrait correlation with the BFI (see Table 2) at moderate to large effect sizes, confirming H2. The same was the case with correlations based on MAP estimates. 
To compare the validity of BFT-MFC and BFT-RS controlling for response format (H3 and H4), we analyzed data from two sources: other-ratings on BFT-MFC and BFT-RS, and self-ratings on HEXACO-MFC and HEXACO-RS. To test H3, that MFC trait estimates would show similar correlations as RS trait estimates on the same or similar constructs when the response format was the same, we first compared monotrait correlations between self- and other-ratings on the BFT when both raters filled out the same version. For all Big Five domains, correlations were larger for BFT-MFC than for BFT-RS at small (conscientiousness: $\left.r_{\mathrm{BFT}-\mathrm{MFC}}=.77, r_{\mathrm{BFT}-\mathrm{RS}}=.69\right)$ to large $\left(\right.$ extraversion: $\left.r_{\mathrm{BFT}-\mathrm{MFC}}=.89, r_{\mathrm{BFT}-\mathrm{RS}}=.73\right)$ effect sizes (see Table 2 and Figure 3). Second, we compared correlations between BFT and HEXACO traits (both self-ratings, same response format). Here, some correlations did not differ between BFT-MFC and BFT-RS (e.g., BFT openness with HEXACO openness correlated .82 with the MFC versions and .79 with the RS versions), while others showed small to medium differences with larger correlations for RS (see Table 2 and Figure 4). Thus, overall and contradicting $\mathrm{H} 3$, we mostly did not find similar correlations. In the majority of cases with at least small differences, the correlation was larger for BFT-MFC than for BFT-RS. Note that when testing this hypothesis with correlations based on MAP estimates, fewer differences were found and the correlations that differed were larger for BFT-RS than BFT-MFC (see Table S2).

H4 also considers correlations between the same or similar traits, but for nonmatching response formats (i.e., heteromethod correlations). Monotrait correlations between self- and other-ratings on the BFT when the two raters filled out different versions (i.e., the self-rater BFT-MFC and the other-rater BFT-RS or in reverse) mostly did not show any differences. In contrast, correlations between BFT and HEXACO (both self-ratings, mismatched response format) on similar traits differed between BFT-MFC and BFT-RS for four of the Big Five (see Table 2). With MAP estimates, only two out of the 10 correlations differed between BFT-MFC and BFT-RS (see Table S2). Furthermore, we compared 
monotrait-monomethod (same response format) with monotrait-heteromethod (different response format) correlations. Here, the majority (16 out of 20) of the model-based correlations differed with almost all of the differences going in the direction of larger monomethod correlations (see Table S3). A similar result was found for correlations based on MAP estimates (see Table S4). Thus, the evidence for H4 is mixed.

In sum, convergent validity coefficients yielded mixed results for the comparison between BFT-MFC and BFT-RS. When looking at the same or similar traits assessed with the same or mismatched response format (other-rater BFT and HEXACO), six out of 20 modelbased correlations did not differ between BFT-MFC and BFT-RS and six only showed small differences, as expected, while the remaining eight showed moderate to large differences. However, with MAP estimates, most correlations (13 out of 20) showed negligible differences.

Discriminant validity. In $\mathrm{H} 5$, we predicted that discriminant validities would be similar between BFT-MFC and BFT-RS when the response format was the same. This was true for $8(17 \%)$ of the correlations, such as the correlation between extraversion in the selfrated BFT and openness in the other-rated BFT $\left(r_{\mathrm{BFT}-\mathrm{MFC}}=.31, r_{\mathrm{BFT}-\mathrm{RS}}=.29\right.$; see Table $\mathrm{S} 5$ and Figure 3). However, the other correlations differed between BFT-MFC and BFT-RS, though most of those differences were small (51\%). For example, extraversion in the selfrated BFT correlated .47 with agreeableness in the other-rated BFT for BFT-MFC, but .33 for BFT-RS. In several cases, the correlations for BFT-MFC and BFT-RS showed opposite signs such as the correlation between neuroticism in the self-rated BFT and openness in the otherrated BFT $\left(r_{\mathrm{BFT}-m a t c h e d}=.33, r_{\mathrm{BFT}-\mathrm{RS}}=-.27\right)$. For correlations with the same sign, almost all BFT-MFC correlations were larger in magnitude than BFT-RS correlations. With MAPs, the results were similar, except that there was no systematic pattern in the direction of the difference (see Table S6). Thus, H5 was not confirmed. 
To test H6, we compared discriminant validity correlations of BFT-MFC and BFT-RS with long and short measures of the Dark Triad, where the response format was only the same for BFT-RS. Fourteen (13 for MAPs) of the 30 correlations did not differ between BFT-MFC and BFT-RS (see Table S5, Table S6, and Figure S4). For example, the model-based correlation between extraversion and narcissism in the Narcissistic Admiration and Rivalry Questionnaire was .35 for BFT-MFC and .29 for BFT-RS. Sixteen correlations showed small to moderate differences such as the correlation between neuroticism and psychopathy in the Self-report Psychopathy Scale $\left(r_{\mathrm{BFT}-\mathrm{matched}}=-.32, r_{\mathrm{BFT}-\mathrm{RS}}=-.12\right)$. However, these correlations were not systematically lower for BFT-MFC, disconfirming H6.

In sum, our hypotheses on discriminant validity coefficients were not confirmed. In many cases correlations differed between BFT-MFC and BFT-RS, even when the same response format was used for both traits. In addition, both for shared and non-shared response formats, BFT-MFC correlations were often larger in magnitude than BFT-RS correlations.

\section{Integrative summary of findings on construct validity}

In sum, our MTMM analysis across hypotheses 1 to 6 showed that when the response format was the same, correlations between the same or similar traits were larger than with different response formats for both BFT-MFC and BFT-RS. The BFT-MFC showed a better correspondence between self- and other-ratings than the BFT-RS with model-based correlations. For both BFT-MFC and BFT-RS, monotrait-heteromethod correlations were almost always larger than heterotrait-monomethod and heterotrait-heteromethod correlations, though there were more exceptions for BFT-MFC than BFT-RS.

\section{Criterion-related validity}

We obtained ratings on hypothesized relationships between the criteria and the Big Five from five experts. We used the mode across the five raters to inform which regressions of criteria on Big Five traits should be modeled. For all of the social activities criteria, experts expected a relationship with extraversion. For the health variables frequency of smoking and 
number of smoked cigarettes a day, relationships with neuroticism and conscientiousness were expected. Frequency of drinking alcohol was expected to be related to extraversion and conscientiousness, life satisfaction to neuroticism, and exercising regularly to conscientiousness. For the ability-related criteria, experts hypothesized a relation between GPA and conscientiousness, but no relationship with the intelligence test score. Therefore, the latter was not included in the analysis. For the charity criteria (donated blood, charity work, voluntary social year), associations with agreeableness were expected. With respect to the other criteria, the number of times traveled abroad for longer than a month was expected to be related to extraversion and openness, punctuality and having a job to conscientiousness, playing an instrument to openness, and engaging in extreme sports to extraversion.

$R^{2}$ values ranged from 0 (e.g., voluntary social year, play instrument) to 0.27 (number of parties a month) with an average of 0.07 (see Table 3) for BFT-MFC. For BFT-RS, $R^{2}$ values ranged from 0 (e.g., donated blood, having a job) to 0.54 (life satisfaction) with an average of 0.09 . When life satisfaction, the only criterion that was assessed with an RS instrument, was removed, average $R^{2}$ values were even more similar with 0.06 for BFT-MFC and 0.07 for BFT-RS. With MAP estimates, the average $R^{2}$ value for BFT-MFC was 0.04 and for BFT-RS 0.06. Thus, criterion-related validities were overall very similar between BFTMFC and BFT-RS 3 , indicating that $\mathrm{H} 7$ was not confirmed. Model-based correlations between the Big Five from BFT-MFC and BFT-RS and the 20 criteria are depicted in Figure S5.

\section{Test-retest reliability}

Analyses of the longitudinal measurement invariance of the BFT showed no noninvariant parameters for the MFC version and two noninvariant thresholds for the RS version. Latent regressions of the Big Five at T2 on the Big Five at T1 yielded standardized

\footnotetext{
${ }^{3}$ The criterion number of cigarettes smoked a day was removed from the analysis because of estimation problems in the MFC format which were probably due to the small sample size for this criterion $(N=74)$.
} 
regression coefficients between 0.74 (extraversion) and 0.79 (openness and agreeableness) for BFT-MFC (see Table S7). For BFT-RS, standardized regression coefficients were higher and ranged from 0.93 (conscientiousness) to 1.12 (agreeableness). Thus, test-retest reliabilities were high for both BFT-MFC and BFT-RS, though they descriptively appeared to be larger for BFT-RS on all traits.

\section{Discussion}

The MFC format has gained popularity in recent years, in particular with the development of IRT models that enable deriving normative trait scores (e.g., Brown \& Maydeu-Olivares, 2011; Stark et al., 2005). The MFC format can potentially overcome many drawbacks of the RS format because it eliminates response biases such as extreme response style and acquiescence (Brown, Inceoglu, \& Lin, 2017; Cheung \& Chan, 2002). These response biases cannot occur in the MFC format because respondents compare items without any additional response scale by either ranking the items according to how well they describe them or by choosing the item that describes them the best and the one that describes them the least. Furthermore, the MFC format reduces faking (Cao \& Drasgow, 2019; Christiansen et al., 2005; Jackson et al., 2000). These advantages are relevant to many areas of psychological assessment. For example, in clinical psychology, respondents with personality disorders might be motivated to distort their scores to avoid or obtain a certain diagnosis. The same might be the case in forensic assessments or when job applicants fill out personality questionnaires during the selection process. In these contexts, the MFC format might lead to more accurate inferences than the RS format. The MFC format has already been applied to a variety of constructs, including broad personality traits such as the Big Five (Brown \& Maydeu-Olivares, 2011; Lee et al., 2018) and adaptive and maladaptive work- and performance-related dimensions (Brown \& Maydeu-Olivares, 2013; Guenole et al., 2018; Stark et al., 2014; Stark, Chernyshenko, Drasgow, \& White, 2012). This study compared an MFC and an RS version of a Big Five instrument, the Big Five Triplets, with respect to 
construct validity, criterion-related validity, and test-retest reliability. Our study has several strengths that extend previous research on this topic. First, we used a between-subjects design with independent samples to avoid any confounding influences or cross-over effects while previous studies had the same sample fill out both versions. Second, our study compared construct validity between normative scoring of MFC and RS data using other-ratings while previous research almost exclusively relied on self-ratings (for an exception see Brown et al., 2017). This had the advantage of eliminating the influence of common method bias. Third, we obtained monomethod correlations also for the MFC format (e.g., BFT-MFC with HEXACOMFC) while previous studies only used other RS instruments to investigate construct validity. Thus, in our study the comparison between formats was more balanced. Fourth, we used a wide range of criteria that were assessed with different methods such as open-ended responses to questions about the frequency of behaviors and objective measurements such as on the punctuality of arriving to the laboratory session. In contrast, previous research mainly relied on criterion variables assessed with RS which could have led to an overestimation of criterion-related validities for the RS format. Fifth, this is the first study that also investigated the test-retest reliability of scores in the MFC and RS format using Thurstonian modeling for the MFC format, thereby giving us insight into the stability of MFC trait estimates compared to RS trait estimates.

At the most general level, our findings imply that the response format matters and that we cannot assume that different response formats are interchangeable and yield equally good assessments and therefore diagnostic inferences in practice (see also Wetzel \& Greiff, 2018). In the following, we discuss the correspondence between the BFT and the BFI, our results on comparing reliability and construct and criterion-related validity between BFT-MFC and BFT-RS, whether there could be remaining ipsativity in the BFT-MFC, and some limitations of our study and future directions.

\section{Do the Big Five Triplets measure the Big Five?}


The Big Five assessed by the BFT-RS corresponded very well to the BFI Big Five, indicating that our items captured the Big Five traits as they are commonly defined. For BFTMFC, this was also the case for neuroticism, extraversion, openness, and, to a lesser degree, conscientiousness. However, this was not the case for agreeableness, which correlated only .24 between BFT-MFC and the BFI. Since BFT-MFC and BFT-RS consist of the exact same items, this raises the question of why the agreeableness items appeared to measure agreeableness well when presented individually, but did not converge with BFI agreeableness when presented in the MFC format. There are several potential reasons. One is that agreeableness was measured with the least amount of items, seven. In the Thurstonian IRT model, each trait has to be located in the latent trait space from comparisons with all other traits (Brown \& Maydeu-Olivares, 2011). A lower number of items for one trait might have a stronger impact in this setting compared to a single-stimulus format such as the RS format. Another potential reason for this discrepancy is item interactions within the triplets. According to Lin and Brown (2017), item interactions can occur when items in a block differ in their desirability or when the item meaning changes in the context of the other items in the block. The BFT-MFC items were matched regarding their absolute level of social desirability. However, when, for example, three slightly social desirable items are presented together (e.g., I am relaxed most of the time. - I organize my time. - I see the good in people.), their relative desirability may still differ, leading participants to favor certain items in a triplet over others. Furthermore, it is also possible that agreeableness items were interpreted differently when presented together with other items in a triplet compared to when they were presented individually. Nevertheless, it is unclear why this mainly affected the agreeableness items and not items on the other Big Five domains. The questionable construct validity of agreeableness in the BFT-MFC notwithstanding, it should be noted that the reliability of trait estimates on agreeableness in the BFT-MFC was not very much lower than that in the BFT-RS (0.68 in BFT-MFC vs. 0.79 in BFT-RS) and that the criterion-related validities for agreeableness 
overall did not differ between BFT-MFC and BFT-RS. For practical purposes this implies that the meaning of items and constructs may be different when applying an MFC as compared to an RS questionnaire.

\section{Comparing reliability between BFT-MFC and BFT-RS}

Trait estimates from the BFT-MFC showed lower reliabilities than trait estimates from the BFT-RS across all Big Five domains. This was also the case for test-retest reliabilities over a period of six months. Lower reliabilities of estimates from the BFT-MFC compared to BFT-RS are expected because each item in the BFT-RS is rated on a four-point scale and therefore provides three bits of binary information (one for each of the thresholds in the graded response model). In contrast, in the BFT-MFC, each pairwise comparison between the items in a triplet results in a dichotomous outcome indicating which item was preferred, yielding only three bits of binary information per triplet. In addition, information is decreased because the estimation of all traits is interdependent (Brown, 2016; Brown \& MaydeuOlivares, 2011). Thus, in terms of the information provided by each item, the RS format is at an advantage compared to the MFC format. In practice, this implies that to obtain the same level of measurement precision as in the RS format, either more items need to be presented in the MFC format or the number of items in a block needs to be increased (e.g., from triplets to tetrads).

In general, when comparing the MFC and RS format, one has to make a trade-off between using the exact same items versus having the same reliability across formats. In our study, we prioritized the former, which led to improved comparability with respect to content, but at the same time placed the MFC format at a disadvantage with respect to reliability. Nevertheless, in absolute terms, reliabilities of trait estimates in the BFT-MFC were mostly around .80 , which are comparable to those in previous research comparing the formats such as Lee et al. (2018). 
We found that construct validity correlations between the BFT and BFI, HEXACO, or Dark Triad measures often showed differences between the MFC and RS version of the BFT that were above our cut-off for a small difference (|.10| for the difference in the Fisher Ztransformed correlations), though this was less often the case with correlations based on MAPs. Some of these differences were expected, such as when the response format only matched for RS, while others were not, such as when the response format was the same. In a number of cases, BFT-MFC showed larger convergent validities than BFT-RS. This was in particular the case with the model-based monotrait-monomethod correlations between the self-rated BFT and the other-rated BFT. A potential reason for this stronger consistency between self- and other-ratings on BFT-MFC is that no differences in rating scale behavior could occur. For example, with BFT-RS, it is possible that the target person preferred extreme categories while the other-rater avoided extreme categories, thus attenuating the correlation between the two. When comparing monomethod (same response format for both questionnaires) correlations to heteromethod (differing response format for the two questionnaires) correlations, we found larger monomethod correlations for both BFT-MFC and BFT-RS. This result could be attributed to common method bias present in the RS format, but not in the MFC format: Heteromethod correlations might be decreased because RS responses are influenced by rating scale biases in addition to the trait of interest. With selfratings in the RS format (e.g., BFT-RS and HEXACO-RS), stable and systematic interindividual differences in rating scale usage might inflate correlations (Wetzel, Carstensen, \& Böhnke, 2013). With the MFC format, common method bias should not occur and correlations should be more accurate than when correlating MFC with RS.

The larger convergent validity coefficients for BFT-MFC were tempered by larger discriminant validities, which in particular occurred for agreeableness. For example, the monomethod correlation between agreeableness in the BFT-MFC and extraversion in the HEXACO was .71 while it was only .47 for BFT-RS. In combination with several moderate 
to large negative intercorrelations between Big Five domains and some discriminant validities of BFT-RS and BFT-MFC going in different directions, this raises the question whether there was any remaining ipsativity in the BFT-MFC. When scores are ipsative, covariances with a construct or criterion will sum to zero (Clemans, 1966). Thus, any high correlation in one direction has to be compensated for by (high) correlations in the other direction. When scores are ipsative, the average intercorrelation between traits will be negative and can be computed as $r=-1 /(k-1)$, where $k$ equals the number of traits (Clemans, 1966; Hicks, 1970). With five traits, we would therefore expect a mean intercorrelation of -.25 for fully ipsative scores. The mean intercorrelation in the BFT-MFC was -.13 while it was .04 in BFT-RS. The substantially smaller negative mean intercorrelation in the BFT-MFC (i.e., -.13 vs. -.25 ) indicates that there were no severe problems with ipsativity, but it is possible that especially the agreeableness dimension retained some ipsative properties despite using normative scoring. Before drawing inferences from MFC responses in practice, one should thus first examine issues of ipsativity in the scores.

Nevertheless, we found that criterion-related validities were mostly comparable between BFT-MFC and BFT-RS with very similar average $R^{2}$ values. This is noteworthy considering the overall lower reliability of scores in the BFT-MFC, the construct validity issues with agreeableness and - to a lesser extent - conscientiousness in the BFT-MFC, and that a few of the criteria (e.g., life satisfaction) were also assessed with rating scales. Thus, with a comparison between equally construct valid and reliable MFC and RS versions of an instrument, it is conceivable that our hypothesis of higher criterion-related validities for the MFC format would be correct. Previous studies applying normative scoring for MFC data also found similar criterion-related validities for MFC compared to RS data (e.g., Brown \& Maydeu-Olivares, 2013).

Why did our results show less similarity between the MFC and RS versions of an instrument with respect to construct validity than previous studies that also used normative 
scoring for both versions? One potential reason is the difference in study design: Previous studies implemented a within-subjects design with one sample filling out both versions (Brown \& Maydeu-Olivares, 2011; Lee et al., 2018). This could potentially result in more similar correlations between the two versions. In contrast, our study used a between-subjects design with independent samples to avoid any confounding influences or cross-over effects occurring by having the same sample fill out both versions.

\section{Limitations and future directions}

Our comparison of the MFC and RS format was conducted using one specific instrument, namely the BFT. Thus, only further research on other instruments, preferably also measuring other traits than the Big Five, can tell whether some of our results were an idiosyncrasy of the BFT or whether they are generalizable beyond the BFT. For example, it would be interesting to investigate the assessment of maladaptive personality traits such as those included in the Alternative Model of Personality Disorders of the DSM-5 Section III (Krueger et al., 2012; Zachar et al., 2016) or the trait domain qualifiers in the ICD-11's classification of personality disorders (World Health Organization, 2018) with the MFC format. The MFC format requires continuous traits, making it a good fit for dimensional trait models of personality disorders. In particular, recent advancements in the area of normative scoring of MFC data (Brown, 2016) could be used to combine dimensional assessment with categorical classification, while ipsative scoring could not be used for deriving cut-offs for the purpose of classification because ipsative score are only meaningful for intraindividual comparisons. Since the MFC format is less susceptible to intentional distortion (Cao \& Drasgow, 2019), future research could investigate whether assessing personality disorders with the MFC format results in higher accuracy of classification than RS questionnaires when compared with (semi-)structured clinical interviews. Considering that the ICD-11 will be used for coding purposes by all members of the World Health Organization from 2022, it is essential to use measurements that will lead to the most accurate classification. 
We preregistered our hypotheses, which is a strength of this study. However, in retrospect, it would have been useful to formulate some of the hypotheses in a more specific manner. For example, for the hypotheses concerning construct validity, it would have been useful to specify how many differences $>|.10|$ were needed to falsify a hypothesis.

The assessment context of our study was a low-stakes situation. To test the feasibility of applying the MFC format in high-stakes contexts for the individuals such as clinical assessments or personnel selection, studies comparing the MFC and RS format in high-stakes assessments using normative scoring are needed. In addition, in these contexts, biases such as faking or halo effects might be more pronounced. Accordingly, the MFC format might manifest its advantages more clearly. In one recent study on this topic, Brown et al. (2017) showed that interrater agreement on 360-degree feedback in an organizational setting was better when using the MFC format to assess competencies compared with rating scales. Nevertheless, in many contexts, such as research settings or large-scale assessments, the stakes are low for individual participants. For example, most of what we know about the structure and development of personality traits as well as their relations to important life outcomes such as mortality, divorce, and occupational attainment (Roberts, Kuncel, Shiner, Caspi, \& Goldberg, 2007) comes from low-stakes assessments using rating scales. As these results might be confounded with biases pertaining to rating scales, it is important to investigate the validity of trait estimates from the MFC format in low-stakes contexts as well.

While it is sensible and necessary to compare the MFC format to the current standard, rating scales, this can also bias our expectations for the MFC format. For example, if we assume that RS responses are always to some extent influenced by socially desirable responding, while this is controlled for in the MFC format by matching with respect to desirability, it is possible that the constructs we measure in the two formats are not exactly equivalent and thus correlations should be somewhat dissimilar. In line with this, Guenole et al. (2018) concluded that the MFC and the RS version of their instrument measured slightly 
different constructs. Moreover, our expectations regarding criterion-related validity might be influenced by the common use of RS to assess both the construct of interest and the criterion. Thus, future studies could also take into account other response formats and not only compare the MFC format to the RS format (for an overview on response formats in self-report questionnaires see Wetzel \& Greiff, 2018). In addition, it would be interesting to investigate what drives the change in construct between different response formats.

Our test construction was guided by the goal of matching the items within triplets with respect to their social desirability. However, with this goal in mind, it was difficult to achieve balanced scale lengths for all Big Five. For example, openness items tended to be rated as neutral in their social desirability whereas for neuroticism or agreeableness, it is nearly impossible to develop items that are perceived as neutral but still measure the respective constructs. Thus, it is difficult to combine openness, neuroticism, and agreeableness items in triplets. In the BFT-MFC, these difficulties resulted in agreeableness being measured with fewer items than the other traits. This in turn seemed to impair trait estimation in the Thurstonian IRT model. Thus, further research on how to obtain ideal constellations of items that are matched for desirability is needed. Furthermore, guidelines for test construction with the MFC format should be developed in order for the MFC format to become more widespread and easier to implement.

\section{Conclusion}

In conclusion, we found that the Big Five Triplets overall appear to measure the Big Five, though the MFC version had some construct validity issues with the agreeableness domain. Nevertheless, criterion-related validities were comparable between the MFC and the RS version, which is impressive considering the lower reliability of trait scores on the BFTMFC. Thus, the MFC format may be a viable alternative to rating scales once open questions such as how to find ideal item constellations are solved. 


\section{References}

Ashton, M. C., \& Lee, K. (2009). The HEXACO-60: A short measure of the major dimensions of personality. Journal of Personality Assessment, 91(4), 340-345. doi:10.1080/00223890902935878

Back, M. D., Küfner, A. C., Dufner, M., Gerlach, T. M., Rauthmann, J. F., \& Denissen, J. J. (2013). Narcissistic admiration and rivalry: Disentangling the bright and dark sides of narcissism. Journal of Personality and Social Psychology, 105(6), 1013-1037. doi:10.1037/a0034431

Bartram, D. (2007). Increasing validity with forced-choice criterion measurement formats. International Journal of Selection and Assessment, 15(3), 263-272. doi:10.1111/j.14682389.2007.00386.x

Birkeland, S. A., Manson, T. M., Kisamore, J. L., Brannick, M. T., \& Smith, M. A. (2006). A meta-analytic investigation of job applicant faking on personality measures. International Journal of Selection and Assessment, 14(4), 317-335. doi:10.1111/j.14682389.2006.00354.X

Brogden, H. E. (1954). A rationale for minimizing distortion in personality questionnaire keys. Psychometrika, 19(2), 141-148.

Brown, A. (2016). Item response models for forced-choice questionnaires: A common framework. Psychometrika, 81(1), 135-160. doi:10.1007/s11336-014-9434-9

Brown, A., Inceoglu, I., \& Lin, Y. (2017). Preventing rater biases in 360-degree feedback by forcing choice. Organizational Research Methods, 20(1), 121-148. doi:10.1177/1094428116668036 
Brown, A., \& Maydeu-Olivares, A. (2011). Item response modeling of forced-choice questionnaires. Educational and Psychological Measurement, 71(3), 460-502. doi:10.1177/0013164410375112

Brown, A., \& Maydeu-Olivares, A. (2013). How IRT can solve problems of ipsative data in forced-choice questionnaires. Psychol Methods, 18(1), 36-52. doi:10.1037/a0030641

Campbell, D. T., \& Fiske, D. W. (1959). Convergent and discriminant validation by the multitrait-multimethod matrix. Psychological Bulletin, 56(2), 81-105. doi:10.1037/h0046016

Cao, M., \& Drasgow, F. (2019). Does forcing reduce faking? A meta-analytic review of forcedchoice personality measures in high-stakes situations. Journal of Applied Psychology. doi:10.1037/ap10000414

Cheung, M. W.-L., \& Chan, W. (2002). Reducing uniform response bias with ipsative measurement in multiple-group confirmatory factor analysis. Structural Equation Modeling, 9(1), 55-77. doi:10.1207/S15328007SEM0901_4

Christiansen, N. D., Burns, G. N., \& Montgomery, G. E. (2005). Reconsidering forced-choice item formats for applicant personality assessment. Human Performance, 18(3), 267-307. doi:10.1207/s15327043hup1803_4

Christie, R., \& Geis, F. L. (1970). Studies in Machiavellianism. New York: Academic Press.

Clemans, W. V. (1966). An analytical and empirical examination of some properties of ipsative measures. Psychometrikc Monograph No. 14. https://www.psychometricsociety.org/sites/default/files/pdf/MN14.pdf

Cohen, J. (1988). Statistical power analysis for the behavioral sciences. New York: Erlbaum. 
Dantlgraber, M. (2011). Itemselektion nach der Item-Response-Theorie (IRT) bei der Entwicklung eines Persönlichkeitstests [Item selection according to Item Response Theory in the development of a personality test]. (Unpublished master's thesis), University of Konstanz, Konstanz.

Dantlgraber, M. (2015). M-KIT: Modularer Kurzintelligenztest [M-KIT: Modular Short intelligence test] Bern, Switzerland: Hogrefe.

Diener, E., Emmons, R. A., Larsen, R. J., \& Griffin, S. (1985). The Satisfaction with Life Scale. Journal of Personality Assessment, 49(1), 71-75. doi:10.1207/s15327752jpa4901_13

Gignac, G. E., \& Szodorai, E. T. (2016). Effect size guidelines for individual differences researchers. Personality and Individual Differences, 102, 74-78. doi:10.1016/j.paid.2016.06.069

Goldberg, L. R. (1992). The development of markers for the Big-Five factor structure. Psychological Assessment, 4, 26-42.

Goldberg, L. R., Johnson, J. A., Eber, H. W., Hogan, R., Ashton, M. C., Cloninger, C. R., \& Gough, H. G. (2006). The international personality item pool and the future of publicdomain personality measures. Journal of Research in Personality, 40(1), 84-96. doi:10.1016/j.jrp.2005.08.007

Guenole, N., Brown, A. A., \& Cooper, A. J. (2018). Forced-choice assessment of work-related maladaptive personality traits: Preliminary evidence from an application of Thurstonian item response modeling. Assessment, 25(4), 513-526. doi:10.1177/1073191116641181

Hallquist, M. N., \& Wiley, J. F. (2018). MplusAutomation: An R package for facilitating largescale latent variable analyses in Mplus. Structural Equation Modeling, 25(4), 621-638. doi:10.1080/10705511.2017.1402334 
Hare, R. D. (1985). Comparison of procedures for the assessment of psychopathy. J Consult Clin Psychol, 53(1), 7-16. doi:10.1037/0022-006x.53.1.7

Heggestad, E. D., Morrison, M., Reeve, C. L., \& McCloy, R. A. (2006). Forced-choice assessments of personality for selection: Evaluating issues of normative assessment and faking resistance. Journal of Applied Psychology, 91(1), 9-24. doi:10.1037/00219010.91 .1 .9

Hicks, L. E. (1970). Some properties of ipsative, normative, and forced-choice normative measures. Psychological Bulletin, 74(3), 167-\&. doi:10.1037/h0029780

Jackson, D. N., Wroblewski, V. R., \& Ashton, M. C. (2000). The impact of faking on employment tests: Does forced choice offer a solution? Human Performance, 13(4), 371388. doi:10.1207/S15327043hup1304_3

John, O. P., Donahue, E. M., \& Kentle, R. L. (1991). The Big Five Inventory-Versions 4 a and 54. Berkeley, CA: University of California, Berkeley, Institute of Personality and Social Research.

John, O. P., \& Srivastava, S. (1999). The Big Five trait taxonomy: History, measurement, and theoretical perspectives. In L. A. Pervin \& O. P. John (Eds.), Handbook of personality theory and research (pp. 102-138). New York: Guilford Press.

Jones, D. N., \& Paulhus, D. L. (2014). Introducing the short Dark Triad (SD3): A brief measure of dark personality traits. Assessment, 21(1), 28-41. doi:10.1177/1073191113514105

Krueger, R. F., Derringer, J., Markon, K. E., Watson, D., \& Skodol, A. E. (2012). Initial construction of a maladaptive personality trait model and inventory for DSM-5. Psychological Medicine, 42(9), 1879-1890. doi:10.1017/S0033291711002674 
Lee, P., Lee, S., \& Stark, S. (2018). Examining validity evidence for multidimensional forced choice measures with different scoring approaches. Personality and Individual Differences, 123, 229-235. doi:10.1016/j.paid.2017.11.031

Lin, Y., \& Brown, A. (2017). Influence of context on item parameters in forced-choice personality assessments. Educational and Psychological Measurement, 77(3), 389-414. doi:10.1177/0013164416646162

McCloy, R. A., Heggestad, E. D., \& Reeve, C. L. (2005). A silk purse from the sow's ear: Retrieving normative information from multidimensional forced-choice items. Organizational Research Methods, 8(2), 222-248. doi:10.1177/1094428105275374

Muthén, L. K., \& Muthén, B. O. (1998-2018). Mplus [Computer software]. Los Angeles, CA: Muthén \& Muthén. Retrieved from www.statmodel.com

Oltmanns, J. R., \& Widiger, T. A. (2018). A self-report measure for the ICD-11 dimensional trait model proposal: The Personality Inventory for ICD-11. Psychological Assessment, 30(2), 154-169. doi:10.1037/pas0000459

Revelle, W. (2018). psych: Procedures for Personality and Psychological Research (Version 1.8.4). Evanston, Illinois: Northwestern University. Retrieved from https://CRAN.Rproject.org/package $=$ psych

Roberts, B. W., Kuncel, N. R., Shiner, R., Caspi, A., \& Goldberg, L. R. (2007). The power of personality The comparative validity of personality traits, socioeconomic status, and cognitive ability for predicting important life outcomes. Perspectives on Psychological Science, 2(4), 313-345. doi:10.1111/j.1745-6916.2007.00047.x

Salgado, J. F., \& Táuriz, G. (2014). The Five-Factor Model, forced-choice personality inventories and performance: A comprehensive meta-analysis of academic and 
occupational validity studies. European Journal of Work and Organizational Psychology, 23(1), 3-30. doi:10.1080/1359432x.2012.716198

Samejima, F. (1969). Estimation of latent ability using a response pattern of graded scores. In (Psychometric Monograph No.17). Retrieved from http://www.psychometrika.org/journal/online/MN17.pdf

Sass, R., Frick, S., Reips, U. D., \& Wetzel, E. (2018). Taking the test taker's perspective: Response process and test motivation in multidimensional forced-choice versus rating scale instruments. Assessment, Advance online publication. doi:10.1177/1073191118762049

Saville, P., Holdsworth, R., Nyfield, G., Cramp, L., \& Mabey, W. (1993). Occupational Personality Questionnaires: Concept model manual and user's guide. Esher, England: Saville \& Holdsworth Ltd.

SHL. (1997). Customer contact: Manual and user's guide. Surrey, England: SHL Group.

Sisson, E. D. (1948). Forced choice - The new Army rating. Personnel Psychology, 1(3), 365381. doi:10.1111/j.1744-6570.1948.tb01316.x

Stark, S., Chernyshenko, O. S., \& Drasgow, F. (2005). An IRT approach to constructing and scoring pairwise preference items involving stimuli on different dimensions: The multiunidimensional pairwise-preference model. Applied Psychological Measurement, 29(3), 184-203. doi:10.1177/0146621604273988

Stark, S., Chernyshenko, O. S., Drasgow, F., Nye, C. D., White, L. A., Heffner, T., \& Farmer, W. L. (2014). From ABLE to TAPAS: A new generation of personality tests to support military selection and classification decisions. Military Psychology, 26(3), 153-164. doi:10.1037/mil0000044 
Stark, S., Chernyshenko, O. S., Drasgow, F., \& White, L. A. (2012). Adaptive testing with multidimensional pairwise preference items: Improving the efficiency of personality and other noncognitive assessments. Organizational Research Methods, 15(3), 463-487. doi:10.1177/1094428112444611

Team, R. C. (2013). R: A language and environment for statistical computing [Computer software]. Vienna, Austria: R Foundation for Statistical Computing. Retrieved from http://www.R-project.org/

Urbanek, S., \& Horner, J. (2015). Cairo: R graphics device using cairo graphics library for creating high-quality bitmap (PNG, JPEG, TIFF), vector (PDF, SVG, PostScript) and display (X11 and Win32) output (Version 1.5-9). Retrieved from https://CRAN.Rproject.org/package $=$ Cairo

van der Linden, D., Nijenhuis, J. T., \& Bakker, A. B. (2010). The General Factor of Personality: A meta-analysis of Big Five intercorrelations and a criterion-related validity study. Journal of Research in Personality, 44(3), 315-327. doi:10.1016/j.jrp.2010.03.003

Van Vaerenbergh, Y., \& Thomas, T. D. (2013). Response styles in survey research: A literature review of antecedents, consequences, and remedies. International Journal of Public Opinion Research, 25(2), 195-217. doi:10.1093/Ijpor/Eds021

Wei, T., \& Simko, V. (2017). R package "corrplot": Visualization of a Correlation Matrix (Version 0.84). Retrieved from https://github.com/taiyun/corrplot

Wetzel, E., Böhnke, J. R., \& Brown, A. (2016). Response biases. In F. R. L. Leong, B. Bartram, F. Cheung, K. F. Geisinger, \& D. Iliescu (Eds.), The ITC International handbook of testing and assessment (pp. 349-363). New York: Oxford University Press. 
Wetzel, E., Carstensen, C. H., \& Böhnke, J. R. (2013). Consistency of extreme response style and non-extreme response style across traits. Journal of Research in Personality, 47(2), 178-189. doi:10.1016/j.jrp.2012.10.010

Wetzel, E., \& Greiff, S. (2018). The world beyond rating scales - Why we should think more carefully about the response format in questionnaires. European Journal of Psychological Assessment, 34(1), 1-5. doi:10.1027/1015-5759/a000469

Williams, K. M., Nathanson, C., \& Paulhus, D. L. (2003). Structure and Validity of the SelfReport Psychopathy Scale-III in Normal Populations. Paper presented at the 111th annual convention of the American Psychological Association, Toronto, Canada.

World Health Organization. (2018). International classification of diseases for mortality and morbidity statistics (11th Revision). Retrieved from https://icd.who.int/browse11/1-m/en

Zachar, P., Krueger, R. F., \& Kendler, K. S. (2016). Personality disorder in DSM-5: An oral history. Psychological Medicine, 46(1), 1-10. doi:10.1017/S0033291715001543

Zhang, B., Sun, T., Drasgow, F., Chernyshenko, O. S., Nye, C. D., Stark, S., \& White, L. A. (2019). Though forced, still valid: Psychometric equivalence of forced-choice and singlestatement measures. Organizational Research Methods, Advance online publication. doi:10.1177/1094428119836486 
Table 1

Descriptive Statistics for Criterion Variables

\begin{tabular}{lllll}
\hline Measure & M (SD) & Min & Max & N \\
\hline Social activities & & & & \\
Number of Facebook friends & $313.40(245.15)$ & 1 & 3000 & 1083 \\
Attended parties / month & $1.86(1.89)$ & 0 & 16 & 1205 \\
Number dates / month & $2.70(3.55)$ & 0 & 30 & 1203 \\
Number of persons dated / year & $2.83(4.79)$ & 0 & 26 & 1204 \\
\hline Health & $1.99(1.75)$ & 1 & 6 & 1207 \\
Frequency of smoking & $11.51(6.14)$ & 2 & 30 & 147 \\
Number of smoked cigarettes / day & $2.85(0.96)$ & 1 & 5 & 1210 \\
Frequency of drinking alcohol & $4.78(1.17)$ & 1 & 7 & 1198 \\
Life satisfaction & & & & \\
\hline Ability & $1.8(0.57)$ & 1 & 3,6 & 285 \\
GPA & $18.77(4.75)$ & 4 & 31 & 529 \\
Intelligence test score & & & & \\
\hline Other varables & & & \\
\hline
\end{tabular}

Other variables

Number times traveled abroad $>1$

month

$0.88(1.69)$

0

13

1211

\begin{tabular}{lllc}
\hline Dichotomous variables & \% Yes & \% No & N \\
\hline $\begin{array}{l}\text { Social activities } \\
\text { Facebook user }\end{array}$ & 90 & 10 & 1213 \\
\hline $\begin{array}{l}\text { Health } \\
\text { Exercise regularly }\end{array}$ & 71 & 29 & 1215 \\
\hline Charity & 39 & 61 & 1213 \\
Donated blood & & & (continued)
\end{tabular}




\begin{tabular}{llll}
\hline Dichotomous variables & \% Yes & \% No & N \\
\hline Charity work & 33 & 67 & 1215 \\
Voluntary social year & 21 & 79 & 1214 \\
\hline Other variables & & & \\
Punctuality & 86 & 14 & 571 \\
Job & 46 & 54 & 1215 \\
Play instrument & 46 & 54 & 1215 \\
Engage in extreme sports & 5 & 95 & 1213 \\
\hline
\end{tabular}

Note. Number of Facebook friends was analyzed as a log-transformed variable ${ }^{4}$. GPA: In the German grading system, $1=\mathrm{A}$ and $4=\mathrm{D}$ (passing).

\footnotetext{
${ }^{4}$ Prior to log-transforming the variable, we recoded outliers that were most likely typos as missing (e.g., 52052052). In addition, we recoded outliers on attended parties a month, number of persons dated a year, and number of times traveled abroad for longer than a month as 1 above the highest valid value or, if they were very likely typos, as missing.
} 
Table 2

Model-based convergent validity coefficients for BFT-MFC and BFT-RS with significance tests and effect sizes of the differences

\begin{tabular}{|c|c|c|c|c|c|c|c|c|c|}
\hline $\begin{array}{l}\text { Trait and } \\
\text { Method } 1\end{array}$ & Trait and Method 2 & $\begin{array}{l}r \text { BFT- } \\
\text { matched }\end{array}$ & $\begin{array}{l}r \text { BFT- } \\
\text { RS }\end{array}$ & $\begin{array}{l}Z \text { BFT- } \\
\text { matched }\end{array}$ & $\begin{array}{l}Z \text { BFT- } \\
\text { RS }\end{array}$ & $\begin{array}{l}\text { Difference } \\
\text { in } Z \text { - } \\
\text { values }\end{array}$ & Effect size & $\begin{array}{l}\text { Test } \\
\text { statistic } \\
\text { dif- } \\
\text { ference }\end{array}$ & $p$-value \\
\hline BFT N self & BFI N self & 0.90 & 0.96 & 1.45 & 1.96 & -0.51 & large & -8.82 & $<.001$ \\
\hline BFT E self & BFI E self & 0.91 & 0.96 & 1.52 & 1.95 & -0.42 & medium & -7.37 & $<.001$ \\
\hline BFT O self & BFI O self & 0.88 & 0.94 & 1.35 & 1.76 & -0.41 & medium & -7.13 & $<.001$ \\
\hline BFT A self & BFI A self & 0.24 & 0.89 & 0.25 & 1.43 & -1.18 & large & -20.49 & $<.001$ \\
\hline BFT C self & BFI C self & 0.56 & 0.87 & 0.63 & 1.33 & -0.70 & large & -12.15 & $<.001$ \\
\hline BFT N self & BFT N other - same & 0.74 & 0.59 & 0.96 & 0.68 & 0.27 & small & 3.26 & 0.002 \\
\hline BFT E self & BFT E other - same & 0.89 & 0.73 & 1.43 & 0.92 & 0.51 & large & 6.05 & $<.001$ \\
\hline BFT O self & BFT O other - same & 0.73 & 0.49 & 0.93 & 0.53 & 0.40 & medium & 4.82 & $<.001$ \\
\hline BFT A self & BFT A other - same & 0.77 & 0.55 & 1.02 & 0.62 & 0.40 & medium & 4.74 & $<.001$ \\
\hline BFT C self & BFT C other - same & 0.77 & 0.69 & 1.01 & 0.85 & 0.16 & small & 1.95 & 0.06 \\
\hline BFT N self & HEXACO EM self - same & 0.46 & 0.71 & 0.50 & 0.89 & -0.39 & medium & -6.82 & $<.001$ \\
\hline BFT E self & HEXACO EX self - same & 0.79 & 0.91 & 1.07 & 1.51 & -0.44 & medium & -7.72 & $<.001$ \\
\hline BFT O self & HEXACO OP self - same & 0.82 & 0.79 & 1.17 & 1.07 & 0.10 & negligible & 1.70 & 0.094 \\
\hline BFT A self & HEXACO AG self - same & 0.45 & 0.49 & 0.49 & 0.54 & -0.06 & negligible & -0.96 & 0.251 \\
\hline BFT C self & HEXACO CO self - same & 0.95 & 0.93 & 1.81 & 1.64 & 0.18 & small & 3.04 & 0.004 \\
\hline BFT N self & BFT N other - different & 0.64 & 0.59 & 0.76 & 0.68 & 0.08 & negligible & 1.00 & 0.241 \\
\hline BFT E self & BFT E other - different & 0.81 & 0.73 & 1.12 & 0.92 & 0.20 & small & 2.43 & 0.021 \\
\hline BFT O self & BFT O other - different & 0.45 & 0.43 & 0.48 & 0.46 & 0.02 & negligible & 0.25 & 0.387 \\
\hline BFT A self & BFT A other - different & 0.44 & 0.46 & 0.47 & 0.50 & -0.03 & negligible & -0.35 & 0.376 \\
\hline BFT C self & BFT C other - different & 0.70 & 0.63 & 0.87 & 0.74 & 0.12 & small & 1.48 & 0.133 \\
\hline BFT N self & HEXACO EM self - different & 0.84 & 0.17 & 1.22 & 0.17 & 1.05 & large & 18.22 & $<.001$ \\
\hline BFT E self & HEXACO EX self - different & 0.89 & 0.88 & 1.41 & 1.36 & 0.05 & negligible & 0.87 & 0.274 \\
\hline BFT O self & HEXACO OP self - different & 0.62 & 0.51 & 0.73 & 0.56 & 0.17 & small & 2.92 & 0.006 \\
\hline BFT A self & HEXACO AG self - different & 0.28 & 0.55 & 0.29 & 0.62 & -0.33 & medium & -5.72 & $<.001$ \\
\hline BFT C self & HEXACO CO self - different & 0.73 & 0.86 & 0.92 & 1.28 & -0.36 & medium & -6.32 & $<.001$ \\
\hline
\end{tabular}


Note. $\mathrm{BFT}=\mathrm{Big}$ Five Triplets, $\mathrm{N}=$ neuroticism, $\mathrm{E}=$ extraversion, $\mathrm{O}=$ openness, $\mathrm{A}=$ agreeableness, $\mathrm{C}=$ conscientiousness, $\mathrm{EM}=$ emotionality, $\mathrm{EX}=$ extraversion, $\mathrm{OP}=$ openness, $\mathrm{AG}=$ agreeableness vs. anger, $\mathrm{CO}=$ conscientiousness, self $=$ self-ratings, other $=$ other-ratings, same $=$ the same format was used as in the respective BFT version, different $=$ a different format was used than in the respective BFT version. 
Table 3

$R^{2}$ from prediction of criteria by Big Five

\begin{tabular}{|c|c|c|c|}
\hline Criterion & Trait(s) & $\begin{array}{l}R^{2} \\
\text { Multidimensional } \\
\text { forced-choice }\end{array}$ & $R^{2}$ Rating scale \\
\hline Number dates / month & $\mathrm{E}$ & 0.01 & 0.05 \\
\hline Number of persons dated / year & $\mathrm{E}$ & 0.05 & 0.04 \\
\hline $\begin{array}{l}\text { Number times traveled abroad }>1 \\
\text { month }\end{array}$ & $\mathrm{E}, \mathrm{O}$ & 0.01 & 0.06 \\
\hline Attended parties / month & $\mathrm{E}$ & 0.27 & 0.23 \\
\hline Number of Facebook friends (log) & $\mathrm{E}$ & 0.26 & 0.18 \\
\hline GPA & $\mathrm{C}$ & 0.03 & 0.00 \\
\hline Frequency of smoking & $\mathrm{N}, \mathrm{C}$ & 0.05 & 0.04 \\
\hline Frequency of drinking alcohol & $\mathrm{E}, \mathrm{C}$ & 0.12 & 0.12 \\
\hline Facebook user & $\mathrm{E}$ & 0.06 & 0.03 \\
\hline Exercise regularly & $\mathrm{C}$ & 0.01 & 0.01 \\
\hline Donated blood & $\mathrm{A}$ & 0.01 & 0.00 \\
\hline Charity work & A & 0.02 & 0.24 \\
\hline Job & $\mathrm{C}$ & 0.03 & 0.00 \\
\hline Instrument & $\mathrm{O}$ & 0.00 & 0.06 \\
\hline Voluntary social year & $\mathrm{A}$ & 0.00 & 0.02 \\
\hline Engage in extreme sports & $\mathrm{E}$ & 0.06 & 0.03 \\
\hline Punctuality & $\mathrm{C}$ & 0.01 & 0.00 \\
\hline Life satisfaction & $\mathrm{N}$ & 0.20 & 0.54 \\
\hline
\end{tabular}

Note. Number of cigarettes a day was removed due to estimation problems. $\mathrm{N}=$ neuroticism, $\mathrm{E}=$

extraversion, $\mathrm{O}=$ openness, $\mathrm{A}=$ agreeableness, $\mathrm{C}=$ conscientiousness 
Please rank the statements according to how well they describe you from most like you (1) to least like you (3).
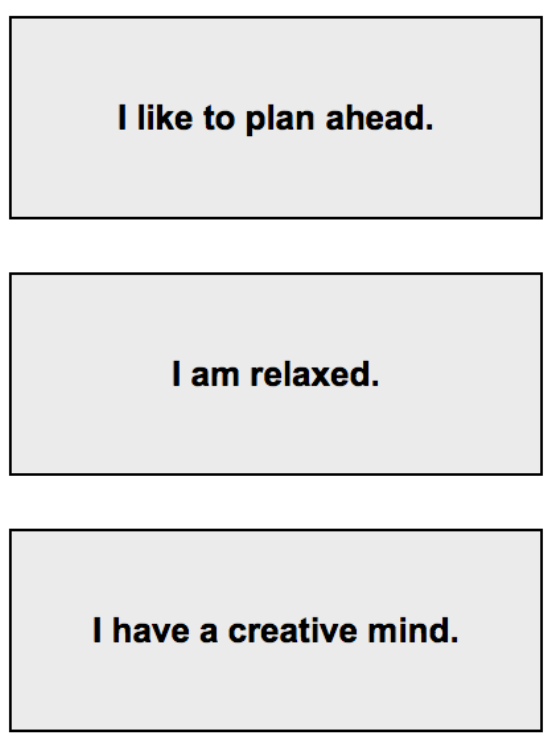

Figure 1. Sample triplet from the Big Five Triplets

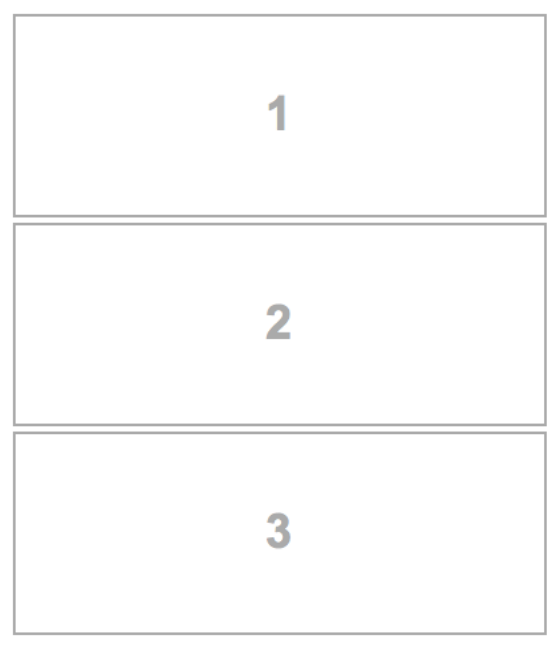




\begin{tabular}{|c|c|c|c|c|c|c|c|c|c|c|}
\hline & \multicolumn{5}{|c|}{ BFT-MFC } & \multicolumn{5}{|c|}{ BFT-RS } \\
\hline & $\mathrm{N}$ & $E$ & 0 & A & C & $\mathrm{N}$ & $E$ & 0 & A & C \\
\hline Neuroticism & 0.9 & -0.19 & $\square$ & -0.64 & 0.29 & 0.96 & -0.3 & - & -0.17 & \\
\hline Extraversion & -0.16 & 0.91 & 0.14 & 0.29 & -0.38 & -0.38 & 0.96 & 0.31 & 0.41 & 口 \\
\hline nventory Openness & 口 & 0.15 & 0.88 & $\square$ & -0.31 & -0.13 & 0.34 & 0.94 & 0.39 & $\square$ \\
\hline Agreeableness & 0.11 & 口 & $\square$ & 0.24 & -0.47 & -0.4 & 0.37 & 0.28 & 0.89 & 0.11 \\
\hline Conscientiousness & -0.13 & -0.11 & $\square$ & $\square$ & 0.56 & -0.24 & 0.17 & 0.11 & 0.26 & 0.87 \\
\hline
\end{tabular}

Figure 2. Multitrait-multimethod correlation matrix between the Big Five Inventory and the Big Five Triplets (BFT) in the multidimensional forced-choice (MFC) and rating scale (RS) version. The size of the square indicates the magnitude of the correlation. Positive correlations are depicted in green and blue and negative correlations in orange and red (refer to online version of article where the figure is in color). Only correlation coefficients $>|.10|$ are shown. $\mathrm{N}=$ neuroticism, $\mathrm{E}=$ extraversion, $\mathrm{O}=$ openness, $\mathrm{A}=$ agreeableness, $\mathrm{C}=$ conscientiousness. 


\begin{tabular}{|c|c|c|c|c|c|c|c|c|c|c|}
\hline & & & BFT- & MFC & & & & BFT. & RS & \\
\hline & $\mathbf{N}$ & E & 0 & A & C & $\mathbf{N}$ & E & 0 & A & C \\
\hline Neuroticism & 0.74 & -0.22 & 0.37 & 0.9 & 0.16 & 0.59 & -0.18 & $\square$ & -0.29 & 口 \\
\hline Extraversion & -0.44 & 0.89 & ш & 0.58 & -0.33 & -0.37 & 0.73 & 0.29 & 0.38 & -0.11 \\
\hline Openness & 0.33 & 0.31 & 0.73 & -0.33 & -0.26 & -0.27 & 0.29 & 0.48 & 0.26 & 口 \\
\hline Agreeableness & -0.56 & 0.47 & -0.21 & 0.77 & -0.27 & -0.4 & 0.32 & 0.27 & 0.55 & . \\
\hline onscientiousness & 0.22 & -0.23 & -0.16 & -0.2 & 0.77 & 口 & $\square$ & $\square$ & 0.11 & 0.69 \\
\hline
\end{tabular}

Figure 3. Multitrait-multimethod correlation matrix between the self-rated Big Five Triplets (BFT) and the other-rated BFT in the multidimensional forced-choice (MFC) and rating scale (RS) version. The target person and the other-rater filled out the same version of the BFT. The size of the square indicates the magnitude of the correlation. Positive correlations are depicted in green and blue and negative correlations in orange and red (refer to online version of article where the figure is in color). Only correlation coefficients $>|.10|$ are shown. $\mathrm{N}=$ neuroticism, $\mathrm{E}$ $=$ extraversion, $\mathrm{O}=$ openness, $\mathrm{A}=$ agreeableness, $\mathrm{C}=$ conscientiousness. 


\begin{tabular}{|c|c|c|c|c|c|c|c|c|c|c|}
\hline & \multicolumn{5}{|c|}{ BFT-MFC } & \multicolumn{5}{|c|}{ BFT-RS } \\
\hline & $\mathrm{N}$ & $E$ & 0 & $A$ & C & $\mathrm{N}$ & $E$ & $\mathrm{O}$ & A & $\mathrm{C}$ \\
\hline Emotionality & 0.46 & 0.13 & 0.51 & $\square$ & 0.59 & 0.71 & $\square$ & 0.17 & 0.33 & 0.24 \\
\hline Extraversion & -0.86 & 0.79 & 0.24 & 0.71 & -0.52 & -0.69 & 0.91 & 0.25 & 0.47 & $\square$ \\
\hline Openness & -0.3 & 0.46 & 0.82 & 0.57 & -0.12 & $\square$ & 0.21 & 0.79 & 0.45 & -0.16 \\
\hline Agreeableness & -0.68 & $\square$ & $\square$ & 0.45 & -0.52 & -0.66 & $\square$ & 0.1 & 0.49 & 口 \\
\hline Conscientiousness & 0.73 & -0.54 & -0.22 & -0.7 & 0.95 & $\square$ & $\square$ & $\square$ & $\square$ & 0.93 \\
\hline Honesty-Humility & $\square$ & -0.26 & $\square$ & a & 0.22 & $\square$ & $\square$ & 0.11 & 0.56 & \pm \\
\hline
\end{tabular}

Figure 4. Multitrait-multimethod correlation matrix between the HEXACO and the Big Five Triplets (BFT) in the multidimensional forced-choice (MFC) and rating scale (RS) version. The HEXACO was presented in the same response format as the respective BFT version. The size of the square indicates the magnitude of the correlation. Positive correlations are depicted in green and blue and negative correlations in orange and red (refer to online version of article where the figure is in color). Only correlation coefficients $>|.10|$ are shown. $\mathrm{N}=$ neuroticism, $\mathrm{E}=$ extraversion, $\mathrm{O}=$ openness, $\mathrm{A}=$ agreeableness, $\mathrm{C}=$ conscientiousness. 